\title{
INTERVENÇÃO DO ENFERMEIRO NA PREVENÇÃO DE TROMBOSE VENOSA PROFUNDA NO PÓS- OPERATÓRIO: REVISÃO INTEGRATIVA
}

\author{
Nurse's Intervention in the Prevention of Deep Venous Thrombosis in the Postoperative Period: Integrative \\ Review \\ Intervención del enfermero en la prevención de la trombosis venosa profunda en el postoperatorio: revisión \\ integradora
}

\author{
Alexandre Rodrigues, ${ }^{*}$ Ana Filipa Advins, ${ }^{* *}$ Ana Rebelo, ${ }^{* * *}$ Cláudia Dias, ${ }^{* * * *}$ Ricardo Novo, ${ }^{* * * * *}$ \\ Sara Anjos, ${ }^{* * * * *}$ António Garrido ${ }^{* * * * * *}$
}

\section{RESUMO}

Enquadramento: a Trombose Venosa Profunda (TVP) carateriza-se pela formação de um coágulo numa veia profunda e pela sua obstrução. As intervenções cirúrgicas são um fator de risco para o desenvolvimento de TVP, tornando-se a intervenção de Enfermagem importante na prevenção desta condição e, por inerência, na diminuição da morbilidade e mortalidade. Objetivo: identificar intervenções de Enfermagem que visam a prevenção de TVP no pós-operatório. Metodologia: revisão Integrativa da Literatura orientada pela questão de investigação: "Qual a intervenção do Enfermeiro na prevenção da TVP em pessoas adultas e idosas, no período pós-operatório?". Após a definição dos critérios de inclusão e exclusão, a pesquisa dos artigos realizou-se nas bases de dados Biblioteca Virtual em Saúde, Pubmed e B-on. Resultados: a seleção totalizou 5 artigos cujos resultados englobam 5 áreas de intervenção em enfermagem: educação para a saúde, promoção precoce da autonomia, potenciamento do retorno venoso, promoção da evolução dietética e profilaxia farmacológica anti-trombótica. Conclusão: o Enfermeiro intervém na prevenção de TVP através da realização de ensinos sobre mobilização precoce, de exercícios respiratórios profundos e de estimulação da tosse, de massagem dos membros inferiores, da aplicação de meias de compressão e da administração de terapia anti trombótica.

Palavras-chave: trombose venosa; cuidados de enfermagem; período pós-operatório

"PhD, Professor Adjunto na Universidade de Aveiro https://orcid.org/0000-0001-8408 $769 \mathrm{X}$ autor de correspondência: alexandre.rodrigues@ua.pt

${ }^{* *}$ RN, Enfermeira, Universidade de Aveiro https://orcid.org/0000-0003-4430-0980

${ }^{* * *}$ RN, Enfermeira, Universidade de Aveiro https://orcid.org/0000-0003-2511-0567

${ }^{* * * *}$ RN, Enfermeira, Universidade de Aveiro https://orcid.org/0000-0001-8933-6261

${ }^{* * * * *}$ RN, Enfermeiro, Santa Casa da Misericórdia de Aveiro https://orcid.org/0000-0001-9500-110X.

${ }^{* * * * * *}$ RN, Enfermeira, Universidade de Aveiro https://orcid.org/0000-0002-91341956

${ }^{* * * * * *}$ RN, Enfermeiro, Universidade de Aveiro https://orcid.org/0000-0002-40555276

Como referenciar:

Rodrigues, A., Advins, A. F., Rebelo, A., Dias, C., Novo, R., Anjos, S. \& Garrido, A. (2020). Intervenção do enfermeiro na prevenção de trombose venosa profunda no pósoperatório: revisão integrativa. Revista de Investigação em Saúde, 3(2), 87-99. doi.org/10.37914/riis.v3i2.89

Recebido para publicação em: 07/08/2020 Aceite para publicação: 30/11/2020

\section{ABSTRACT}

Background: deep Venous Thrombosis (DVT) is characterized by the formation of a clot in a deep vein and its partial or total obstruction. Surgical interventions are a risk factor for the development of DVT, becoming the Nursing intervention important in the prevention of this condition and, inherently, in reducing morbidity and mortality. Objective: to identify nursing interventions aimed to prevent DVT in the postoperative period. Methodology: Integrative literature review guided by the research question: "What is the nurse's intervention in the prevention of DVT in adult and elderly people, in the postoperative period?". After defining the inclusion and exclusion criteria, the articles were searched in the Biblioteca Virtual em Saúde, Pubmed and B-on databases. Results: the selection totaled 5 articles whose results encompass 5 areas of nursing intervention:-health education, early promotion of autonomy, potentiation of venous return, promotion of dietary evolution and anti-thrombotic pharmacological prophylaxis. Conclusion: nurses are crucial in the prevention of DVT through teaching about early mobilization, deep breathing exercises and coughing, massage of the lower limbs, application of compression stockings and administration of anti-thrombotic therapy.

Keywords: venous thrombosis; nursing care; postoperative period

\section{RESUMEN}

Marco contextual: la Trombosis Venosa Profunda (TVP) se caracteriza por la formación de un coágulo en una vena profunda y por su obstrucción parcial o total. Las intervenciones quirúrgicas son un factor de riesgo para el desarrollo de la TVP, convirtiéndose la intervención de la Enfermería importante en la prevención de esta condición y, de forma inherente, en la reducción de la morbilidad y mortalidad. Objetivo: identificar intervenciones de Enfermería que pretenden prevenir la TVP en el posoperatorio. Metodología: revisión Integrativa de la literatura guiada por la pregunta de investigación: "¿Cuál es la intervención del enfermero en la prevención de la TVP en adultos y ancianos, en el postoperatorio?". Después de definir los criterios de inclusión y exclusión, los artículos fueron buscados en las bases de datos de la Biblioteca Virtual em Saúde, Pubmed y B-on. Resultados: la selección fue de 5 artículos cuyos resultados abarcan 5 áreas de intervención de enfermería: educación en salud, promoción temprana de la autonomía, potenciación del retorno venoso, promoción de la evolución dietética y profilaxis farmacológica antitrombótica. Conclusión: Las enfermeras son cruciales en la prevención de la TVP a través de la enseñanza sobre movilización temprana, ejercicios de respiración profunda y tos, masaje de miembros inferiores, aplicación de calcetines de compresión y administración de terapia antitrombótica.

Palabras clave: trombosis de la vena; atención de enfermería; periodo posoperatorio 


\section{INTRODUÇÃO}

A Trombose Venosa Profunda (TVP) corresponde à formação de um coágulo no interior de um vaso sanguíneo venoso, na sequência de uma reação inflamatória ou trauma, levando à obstrução parcial ou total deste vaso (Pinho, Viegas \& Caregnato, 2016).

Segundo Amaral et al. (2014), a TVP apresenta uma incidência de $25 \%$ em Portugal. Atendendo aos dados da Sociedade Portuguesa de Cirurgia (2015) ocorrem, em Portugal, cerca de 2 mil milhões de casos de TVP por ano, sendo que 60 mil são mortais. A nível internacional, na Alemanha, e de acordo com Heil et al. (2017) a incidência de TVP é de 1 em 1000 pessoas por ano. Nos Estados Unidos da América, Olaf \& Cooney (2017) referem que a partir dos 60 anos há um aumento de cerca de $1 \%$ da probabilidade de desenvolver TVP e que a taxa de recorrência da condição clínica 10 anos após o seu primeiro diagnóstico é de aproximadamente $25 \%$, dependendo, no entanto, da etiologia da trombose. Os mesmos autores indicam que a estimativa de sobrevivência a curto prazo associada à ocorrência de TVP varia entre 95-97\%, enquanto que a longo prazo a mesma estimativa varia entre 61-75\% (Olaf \& Cooney, 2017). Heil et al. (2017), realça que cerca de $35,9 \%$ dos casos de TVP pós-operatória ocorre nos membros inferiores e $53,8 \%$ nos membros superiores.

Dos fatores de risco para a TVP, destacam-se a hospitalização em caso de doença aguda, cancro, idade superior a 60 anos, uso de contraceção oral combinada ou terapia de substituição hormonal, gravidez/pósparto, comportamentos tabágicos, obesidade, viagens superiores a 4 horas (pela diminuição da mobilidade) e cirurgia, apresentando este último um risco 70 vezes superior para desenvolver uma TVP (Macneill \& Bagot,
2018). Este risco pode estar relacionado com a manipulação cirúrgica, e consequentes lesões tecidulares nas veias, que leva à libertação de substâncias vasoativas, de produtos pró-inflamatórios e de fatores coagulantes pelos tecidos do local da cirurgia, atuando de forma local e sistémica, aumentando o risco trombogénico (Sociedade Portuguesa de Cirurgia, 2015).

Atendendo ao referido por Di Nisio, van Es \& Büller (2016), para além dos fatores suprarreferidos, fazem parte dos fatores de risco os antecedentes pessoais ou familiares de Tromboembolismo Venoso (TEV), doenças autoimunes e doenças inflamatórias crónicas, trauma ou fratura, presença de cateter venoso central ou pacemaker e situações de paresia ou paralisia.

Assume-se, assim, que a intervenção autónoma e interdependente do Enfermeiro se revela importante tendo em vista a minimização destes fatores de risco, especialmente no que se refere à promoção, manutenção e recuperação da saúde do paciente submetido a cirurgia. Nesta lógica, é de igual forma relevante referir que a intervenção e os resultados obtidos no pós-operatório se sustentam numa intervenção de enfermagem programada e estruturada que se inicia no pré-operatório. Estes pressupostos vão ao encontro do que é defendido pela Associação dos Enfermeiros de Sala de Operações Portugueses (AESOP, 2012), quando refere que a função do enfermeiro é identificar as necessidades do doente e sua família para que possa deste modo organizar e implementar um plano individualizado de cuidados de enfermagem, baseado no conhecimento das ciências humanas e da natureza, conduzindo ao restabelecimento ou conservação da saúde e bemestar do indivíduo nas fases pré, intra e pós 
operatórias. O período pós-operatório é o período que se segue após o término da cirurgia e se estende até à alta clínica. Caracteriza-se por ser um momento de instabilidade funcional, durante o qual podem surgir complicações, implicando a verificação das repercussões orgânicas e fisiológicas e a implementação de cuidados específicos, com o objetivo de promover a restituição dos processos fisiológicos, pela melhoria na função, resolução da sintomatologia adversa e o retorno ao estado prévio à cirurgia (Bower \& Royse, 2016; Lopes et al., 2019). Desta forma, a intervenção do Enfermeiro é importante para dar continuidade ao trabalho desenvolvido no pré e intra-operatório, no sentido de promover a recuperação e prevenir as complicações pós-operatórias.

É através de intervenções autónomas e interdependentes, que a enfermagem demonstra capacidades para atuar na linha da frente no pósoperatório, prevenir essas complicações e, por inerência, reduzir o tempo de permanência hospitalar, a taxa de mortalidade e evitar a cirurgia de repetição (Sousa et al., 2020).

Mundialmente realizam-se aproximadamente 234,2 milhões de procedimentos cirúrgicos, dos quais 7 milhões resultam em complicações evitáveis. Este facto constitui um problema de saúde pública, requerendo um especial foco da intervenção de Enfermagem (Sousa et al., 2020). Considerando este e outros dados epidemiológicos, torna-se importante para a Enfermagem a procura de evidência científica, que permita a criação de protocolos de avaliação e intervenção que possibilitarão a melhoria da prática de enfermagem, no sentido de a tornar mais direcionada e rigorosa (Lopes et al., 2019).
Atendendo à problemática em estudo, formulou-se como questão de investigação "Qual a intervenção do Enfermeiro na prevenção da TVP em pessoas adultas e idosas no período pós-operatório?". A partir da questão de investigação, definimos como objetivo: identificar as intervenções de Enfermagem que visam a prevenção de TVP no período pós-operatório.

\section{PROCEDIMENTOS METODOLÓGICOS DE REVISÃO}

A presente Revisão Integrativa da Literatura (RIL) foi orientada através da questão de investigação "Qual a intervenção do Enfermeiro na prevenção da TVP em pessoas adultas e idosas no período pós-operatório?". A questão foi formulada utilizando a abordagem “PICO" cujo acrónimo representa: Patient (utente), Intervention (intervenção), Comparison (comparação) e Outcome(s) (resultados). Tal como é apresentado por Sousa et al. (2017), esta abordagem pode ser contemplada como método numa RIL de forma a integrar os elementos essenciais na questão de investigação. Assim, definiu-se que: população pessoas adultas e idosas submetidas a uma cirurgia; intervenções - intervenção do Enfermeiro no período pós-operatório; comparação - não se aplica nesta revisão; resultados - prevenção da TVP.

De forma a uniformizar os conceitos de pesquisa, foram identificados os seguintes Descritores em Ciências da Saúde (DeCS): Trombose Venosa/Venous Thrombosis/Trombosis de la Vena; Assistência Perioperatória/Perioperative Care/Atención Perioperativa; Cuidados de Enfermagem/Nursing Care/Atención de Enfermería; Período Perioperatório/Perioperative Period/Periodo Perioperatorio.

Para a pesquisa foram utilizados os descritores em 
inglês e formuladas as seguintes frases booleanas:

"“venous thrombosis" AND "perioperative care" AND

"nursing care"” e "“venous thrombosis" AND

"perioperative period" AND "nursing care"”". A pesquisa foi realizada entre 1 e 31 de março de 2020 nas seguintes bases de dados: Biblioteca Virtual em Saúde, Pubmed e B-on. Os resultados da pesquisa descrita são apresentados na Tabela 1.

Tabela 1

Resultados das pesquisas nas bases de dados por frase booleana

\begin{tabular}{c|c|c|c}
\hline Pesquisa & Biblioteca Virtual em Saúde & Pubmed & B-on \\
\hline $\begin{array}{c}\text { "venous thrombosis" AND "perioperative } \\
\text { care" AND "nursing care" }\end{array}$ & 11 & 1 & 1245 \\
\hline $\begin{array}{c}\text { "venous thrombosis" AND "perioperative } \\
\text { period" AND "nursing care" }\end{array}$ & 3 & 1 & 1676 \\
\hline
\end{tabular}

Os critérios de inclusão definidos para o estudo foram relativos ao espaço temporal da publicação dos estudos (entre janeiro de 2010 e dezembro de 2019), ao idioma (português, espanhol e inglês), à disponibilidade do artigo integral, à tipologia do estudo (estudos primários), à população alvo (adultos e idosos submetidos a cirurgia), à intervenção (cuidados de Enfermagem e o período pós-operatório), resultado esperado (a prevenção da TVP). Os critérios de exclusão utilizados dizem respeito à duplicação de artigos e a artigos em que se abordem exclusivamente intervenções de outras classes profissionais. Foi ainda considerada a avaliação da qualidade dos artigos com pontuação igual ou superior a 0,55 na escalas desenvolvidas por Kmet, Lee \& Cook (2004), visto que segundo Sousa et al. (2017) os estudos a integrar numa RIL devem ser analisados com detalhe e de forma rigorosa, com o intuito de garantir a validade da revisão.
O processo de pesquisa e de seleção dos artigos foi realizado conforme está explícito no fluxograma da Figura 1. De ressalvar que o processo de pesquisa foi efetuado por dois grupos de revisores independentes - sendo um grupo constituído por dois elementos e outro por três - e que estes obtiveram o mesmo número final de artigos. Após a extração dos artigos das bases de dados, o processo de seleção incluiu os critérios de inclusão de ano de publicação, de idioma, de duplicação e de texto integral do artigo, que foram aplicados ao título. Os critérios da tipologia do estudo e da inclusão das temáticas dos cuidados de Enfermagem e do período pós-operatório foram analisados na leitura do resumo. Os restantes critérios foram aplicados ao texto integral. No final do processo, foi aplicado o instrumento de avaliação da qualidade acima referido, o que garantiu a qualidade dos artigos dentro dos parâmetros definidos. 
Intervenção do enfermeiro na prevenção de trombose venosa profunda no pós-operatório: revisão integrativa

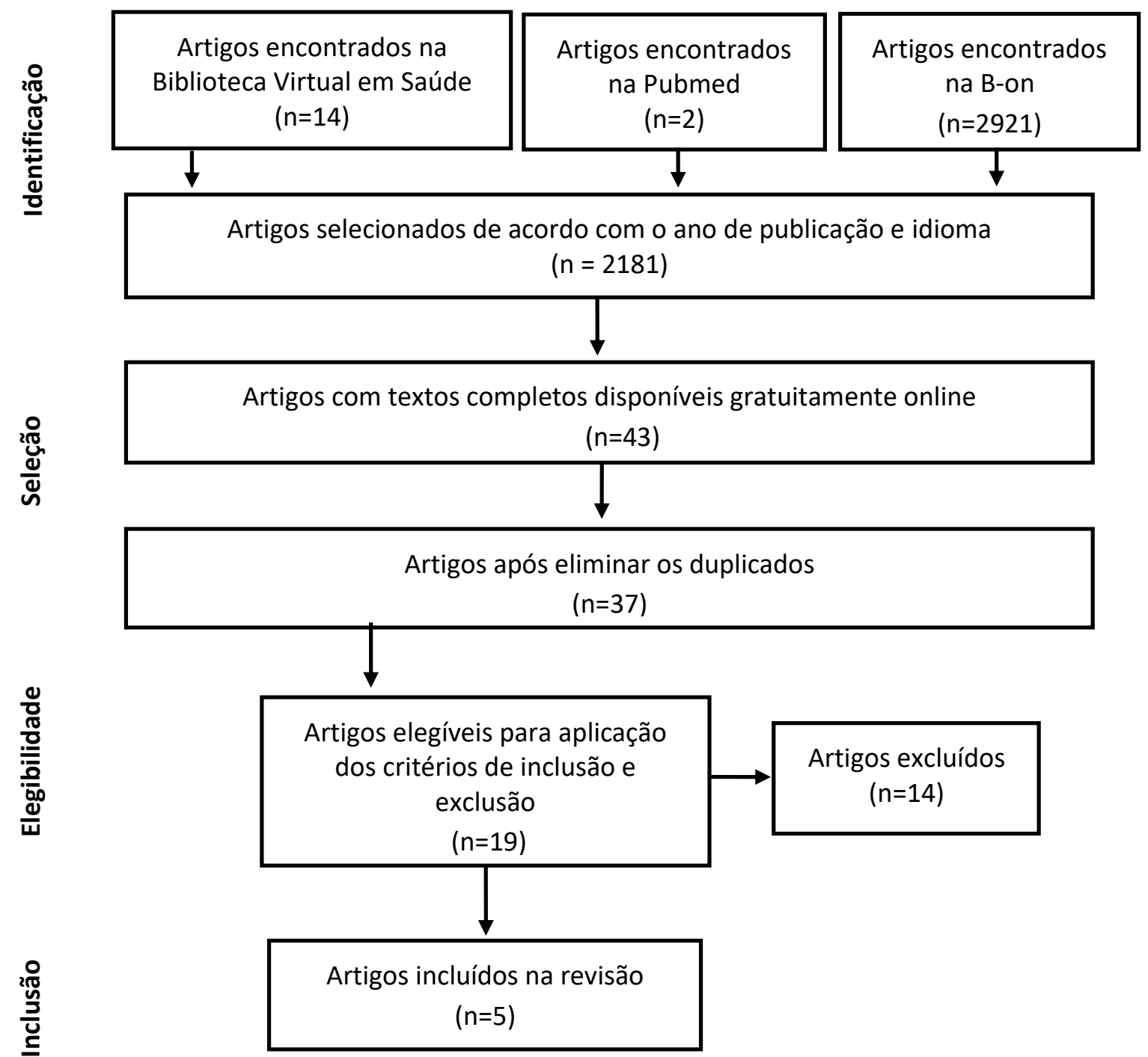

Figura 1

Fluxograma do processo de seleção, segundo PRISMA.

\section{RESULTADOS}

Após a aplicação dos critérios, a amostra final integrou os seguintes 5 artigos: Kang et al. (2019), Yayla \& Özer (2019), Jia et al. (2013), Pu, Jiang, Leng \& Yang (2017) e Ghelase et al. (2013). Da análise dos estudos obtidos, apresentam-se os resultados à luz da questão de investigação inicial e dos objetivos da presente revisão. Para um melhor entendimento, inicialmente apresentam-se os dados caraterizadores dos estudos, na

Tabela 2, seguindo-se a exposição das intervenções de Enfermagem para a prevenção da TVP descritas em cada um dos estudos. 
Tabela 2

Caraterização geral dos estudos

\begin{tabular}{|c|c|c|c|}
\hline Autor/Ano & Tipos de estudos & Objetivos & Conclusões \\
\hline Kang et al./ 2019 & $\begin{array}{l}\text { Estudo quase-experimental } \\
\text { com grupo de controlo } \\
\text { Epidemiologia retrospetiva } \\
\text { e prospetiva }\end{array}$ & $\begin{array}{l}\text { Avaliar o efeito do protocolo } \\
\text { ERAS no período } \\
\text { perioperatório em pacientes } \\
\text { submetidos a colocação de } \\
\text { prego femoral proximal anti- } \\
\text { rotação por fratura } \\
\text { intertrocantérica. }\end{array}$ & $\begin{array}{l}\text { A implementação do protocolo ERAS } \\
\text { nos pacientes submetidos a cirurgia por } \\
\text { fratura intertrocantérica reduz } \\
\text { significativamente o tempo de } \\
\text { permanência hospitalar sem } \\
\text { comprometer a recuperação funcional } \\
\text { precoce do paciente. }\end{array}$ \\
\hline Yayla \& Özer/ 2019 & $\begin{array}{l}\text { Estudo quase-experimental } \\
\text { com grupo de controlo } \\
\text { Epidemiologia prospetiva }\end{array}$ & $\begin{array}{l}\text { Determinar o efeito de uma } \\
\text { mobilização precoce no } \\
\text { período pós-operatório em } \\
\text { pacientes submetidos a } \\
\text { cirurgia cardíaca. }\end{array}$ & $\begin{array}{l}\text { Este estudo indica que a mobilização } \\
\text { precoce na UTI e cirurgia de clínica } \\
\text { cardíaca é eficaz na redução de } \\
\text { complicações no período pós- } \\
\text { operatório e no tempo de permanência } \\
\text { hospitalar e na melhoria da pontuação } \\
\text { da Richards Campbell Sleep } \\
\text { Questionnaire (RCSQ). }\end{array}$ \\
\hline Jia et al./ 2013 & $\begin{array}{l}\text { Estudo controlado } \\
\text { randomizado } \\
\text { Epidemiologia prospetiva }\end{array}$ & $\begin{array}{l}\text { Estudar a probabilidade da } \\
\text { Fast-track surgery (cirurgia } \\
\text { por via rápida) evitar ou } \\
\text { reduzir a ocorrência de } \\
\text { delírio pós-operatório, assim } \\
\text { como outras complicações } \\
\text { em pacientes idosos com } \\
\text { carcinoma colorretal, e } \\
\text { avaliar a função de IL-6 no } \\
\text { delírio pós-operatório. }\end{array}$ & $\begin{array}{l}\text { Fast-track surgery (cirurgia por via } \\
\text { rápida) diminui o tempo de } \\
\text { permanência hospitalar, facilita a } \\
\text { recuperação dos movimentos } \\
\text { intestinais, e reduz a ocorrência de } \\
\text { delírio pós-operatório e outras } \\
\text { complicações em pacientes idosos com } \\
\text { carcinoma colorretal. }\end{array}$ \\
\hline $\begin{array}{l}\text { Pu, Jiang, Leng \& Yang / } \\
2017\end{array}$ & $\begin{array}{l}\text { Estudo controlado não } \\
\text { randomizado } \\
\text { Epidemiologia prospetiva }\end{array}$ & $\begin{array}{l}\text { Investigar a eficácia clínica } \\
\text { da intervenção precoce de } \\
\text { Enfermagem na TVP em } \\
\text { pacientes senis com fratura } \\
\text { traumática durante o } \\
\text { período perioperatório. }\end{array}$ & $\begin{array}{l}\text { A intervenção precoce de Enfermagem } \\
\text { em pacientes senis com fratura } \\
\text { traumática durante o período } \\
\text { perioperatório diminuiu a taxa de } \\
\text { incidência de TVP eficazmente, assim } \\
\text { como aliviar a dor e melhorar a relação } \\
\text { médico-paciente. }\end{array}$ \\
\hline Ghelase et al./ 2013 & $\begin{array}{l}\text { Estudo descritivo } \\
\text { Epidemiologia prospetiva }\end{array}$ & $\begin{array}{l}\text { Realizar uma avaliação } \\
\text { preoperatória dos fatores de } \\
\text { risco de trombose num } \\
\text { grupo de pacientes } \\
\text { submetidos a profilaxia pré- } \\
\text { operatória com Heparina de } \\
\text { Baixo Peso Molecular } \\
\text { (HBPM) associada a outras } \\
\text { medidas de prevenção. }\end{array}$ & $\begin{array}{l}\text { Cada paciente submetido a intervenção } \\
\text { cirúrgica deve ser avaliado para } \\
\text { qualquer fator de risco que possa } \\
\text { provocar um evento tromboembólico. } \\
\text { Os cuidados gerais de Enfermagem e as } \\
\text { estratégias profiláticas com HBPM são } \\
\text { decididos consoante esses fatores. }\end{array}$ \\
\hline
\end{tabular}

Numa análise global, os estudos de Kang et al. (2019), Yayla \& Özer (2019), Jia et al. (2013) e Pu, Jiang, Leng \& Yang (2017) apresentam uma comparação entre duas abordagens distintas de intervenções de Enfermagem, sendo que uma é a abordagem padronizada nas diferentes unidades de saúde e a outra é definida por diferentes protocolos. $O$ estudo de
Ghelase et al. (2013), difere ligeiramente dos demais, visto que compara os resultados da aplicação ou não de determinado procedimento.

No estudo de Kang et al. (2019) foi analisada aplicação do protocolo ERAS que, no período pós-operatório, tem como princípios: promoção precoce de autonomia; mobilização precoce com recurso a um 
terapeuta de reabilitação; realização de alta clínica segura e com apoio. Os pacientes submetidos ao protocolo experimental foram sujeitos a uma mobilização precoce (<24 horas), sendo que $82 \%$ dos mesmos tiveram alta clínica em 4 dias ou menos, estando a média de dias de permanência hospitalar situada entre os 5 e 7 dias para este grupo. Por outro lado, no grupo de controlo apenas $48 \%$ dos pacientes foram mobilizados dentro de 24 horas após a cirurgia, estando a média de dias de permanência hospitalar situada entre 7 e 9 dias. Foi ainda reportado que as feridas cirúrgicas tiveram uma boa evolução cicatricial sem apresentarem complicações clínicas como infeção, TVP dos membros inferiores e EP, tendo a taxa de incidência destas complicações sido calculada nos primeiros 30 dias pós-cirurgia.

No que respeita ao estudo de Yayla \& Özer (2019), recorreu-se ao protocolo da mobilização precoce que foi elaborado usando o protocolo Cardiac Surgical Fast-Track, outros protocolos de mobilização precoce e resultados de estudos prévios. O protocolo foi aplicado durante 5 dias após a cirurgia e incluía as seguintes intervenções: elevação da cabeceira da cama entre 30 o e 45; uso do espirómetro de incentivo; exercícios de respiração profunda e de tosse; movimentos passivos e ativos dos membros inferiores e superiores; adoção da posição sentada; realização de pequenos percursos de deambulação. De notar que todas as intervenções foram adequadas a cada dia do período pós-operatório, aumentando o número de repetições, de ciclos, de distância e de tempo. Ao aplicar o protocolo de mobilização precoce ao grupo experimental, os autores verificaram que o desenvolvimento de complicações tardias (TVP, pneumonia e/ou disritmia), surgiu em $23,5 \%$ dos participantes. Em relação ao grupo de controlo puderam observar o desenvolvimento das mesmas complicações, em $43,1 \%$ dos participantes. A ocorrência destas complicações foi avaliada através da aplicação da RCSQ aos participantes, em entrevista presencial, nos dias pós-operatórios de 1 a 5 .

No estudo de Jia et al. (2013), foi aplicado o protocolo de Fast-Track Surgery ao grupo experimental. Neste protocolo, na gestão do período pós-operatório é contemplada: ingestão hídrica e alimentar fracionadas; remoção precoce do cateter urinário; mobilização precoce nas primeiras 24 horas. Ao compararem a ocorrência de TVP, documentada com base no Sistema de Classificação Clavien-Dindo, na recuperação pós-operatória, os autores referem não existir uma diferença estatisticamente significativa entre o grupo de controlo e o grupo experimental. No entanto, deve referir-se que no grupo experimental a taxa de incidência de TVP foi de 3,4\%, enquanto que no grupo de controlo foi de $6 \%$.

Relativamente ao estudo de Pu, Jiang, Leng \& Yang (2017), o protocolo implementado no grupo experimental previa que, no período pós-operatório, os Enfermeiros ensinassem os pacientes para a realização de exercícios fora do leito e, se adequado, massajassem os membros inferiores de forma a promover o retorno venoso e reduzir a incidência de TVP. Adicionalmente, segundo indicação médica, poderia ser administrada Nadroparina Cálcica (uma HBPM) ou Ácido Acetilsalicílico (AAS) para profilaxia da condição clínica em causa. Por último, e em pacientes com elevado risco de desenvolvimento de TVP, os Enfermeiros aplicavam meias de compressão ou outros dispositivos para melhorar a eficácia profilática. Neste estudo, observou-se que, após a intervenção de 
Enfermagem, os níveis de Dímero D no sangue ao 4ํe 60 dias após a cirurgia no grupo experimental foram mais baixos em relação aos níveis no grupo de controlo e esta diferença foi estatisticamente significativa. De acordo com a avaliação do nível de Dímero $\mathrm{D}$, no grupo experimental foi reportada uma taxa de incidência de TVP de 9,68\% e no grupo de controlo de $35,48 \%$, tendo os resultados mostrado que houve uma diferença estatisticamente significativa.

Por último, no estudo descritivo de Ghelase et al. (2013) foi implementado um protocolo de administração de HBPM que contemplava a administração da primeira dose até 2 horas antes da cirurgia, da dose seguinte após 12 horas e, segundo este esquema, durante 5-7 dias, numa dosagem recomendada e de acordo com o nível de risco do paciente. Contemplava ainda o prolongamento da heparinoterapia em caso de imobilização ou hospitalização. Após a implementação do protocolo, verificou-se que a incidência de TVP dos membros inferiores foi de 0,92\% e que, na ausência de terapia com heparina, foi de 15-40\%. A avaliação da ocorrência de TVP foi realizada através de investigação laboratorial e realização de ultrassonografia venosa com doppler em caso de suspeita clínica da condição. A Tabela 3 sistematiza as áreas de intervenção de Enfermagem autónomas e interdependentes que emergiram dos estudos analisados, nas quais se discriminam os cuidados específicos a considerar.

Tabela 3

Áreas de Intervenção de Enfermagem

\begin{tabular}{|c|c|}
\hline Áreas de Intervenção & Cuidados de Enfermagem \\
\hline Educação para a saúde & $\begin{array}{l}\text { - Ensinar sobre atividade física (Pu, Jiang, Leng \& Yang, 2017); } \\
\text { - Ensinar sobre alimentação saudável (Pu, Jiang, Leng \& Yang, 2017). }\end{array}$ \\
\hline Adequação do regime dietético & $\begin{array}{l}\text { - Hidratar (primeiras 48h) (Jia et al., 2013). } \\
\text {-Promover dieta fracionada (primeiras 48h) (Jia et al., 2013). }\end{array}$ \\
\hline Promoção precoce da autonomia & $\begin{array}{l}\text { - Mobilizar precocemente (primeiras 24h) - exercícios passivos e ativos (Kang } \\
\text { et al.,2019; Jia et al., 2013; Yayla \& Özer; 2019; Pu, Jiang, Leng \& Yang, 2017); } \\
\text { - Executar exercícios respiratórios profundos e promover a tosse (Yayla \& } \\
\text { Özer; 2019); } \\
\text { - Elevar a cabeceira entre } 30 \text { e 45 (Yayla \& Özer; 2019); } \\
\text { - Posicionar em fowler (na cadeira/cadeirão) por períodos (Yayla \& Özer; } \\
\text { - Remover o cateter urinário (Jia et al., 2013); } \\
\text { - Estimular a deambulação (Yayla \& Özer; 2019). }\end{array}$ \\
\hline Estimulação do retorno venoso & $\begin{array}{l}\text { - Massajar os membros inferiores (Pu, Jiang, Leng \& Yang, 2017); } \\
\text { - Aplicar meias de compressão (Pu, Jiang, Leng \& Yang, 2017). }\end{array}$ \\
\hline Profilaxia tromboembólica & $\begin{array}{l}\text { - Administrar HBPM (Ghelase et al., 2013; Pu, Jiang, Leng \& Yang, 2017); } \\
\text { - Administrar AAS (Pu, Jiang, Leng \& Yang, 2017). }\end{array}$ \\
\hline
\end{tabular}

\section{DISCUSSÃO}

Considerando os resultados obtidos, a discussão será dirigida indo ao encontro das áreas de intervenção de enfermagem na prevenção da TVP no pós-operatório que foram evidenciadas nos estudos encontrados.

A educação para a saúde trata-se de uma área 
transversal a algumas das áreas de intervenção de enfermagem que emergiram neste estudo, daí que se destaque o seu papel na prevenção desta patologia, especialmente no reforço da necessidade de existir um papel ativo por parte do paciente. Segundo Greenall (2016), a falta de conhecimento da própria condição clínica influência negativamente a atitude comportamental que o paciente adota, estando este menos envolvido nos próprios cuidados. Assim, o Enfermeiro deve identificar as barreiras e desenvolver intervenções individualizadas, a fim de o capacitar e aumentar a sua adesão à mudança de comportamentos desajustados, nomeadamente no que respeita à alimentação e ao exercício físico. Estes dois aspetos encontram-se descritos no estudo de $\mathrm{Pu}$, Jiang, Leng \& Yang, 2017 e são reforçados por Barnard (2018) que afirma que os alimentos vegetais não refinados apresentam baixo teor de gordura e alto teor de fibras - o que, associado à prática de exercício físico, melhora a fibrinólise e ajuda a reduzir o risco de TVP. Neste sentido, numa investigação de Guo et al. (2019) comprovou-se que a educação para a saúde desempenha um papel importante especificamente na prevenção de TVP, ao proporcionar aos pacientes uma perceção mais correta sobre a referida condição e sobre as atividades necessárias para melhorar essa mesma condição. Esta conclusão foi possível pela comparação com dados da ocorrência de TVP em pacientes sujeitos à mesma cirurgia anteriormente.

Além das alterações já acima referidas, os pacientes cirúrgicos estão sujeitos a alterações metabólicas e fisiológicas que podem levar ao comprometimento do estado nutricional. A desnutrição ocorre em 22 - 58\% dos casos destes pacientes, tendo um impacto negativo no tempo de permanência hospitalar, na ocorrência de complicações e nos custos hospitalares (Grass et al., 2017; Isidro \& Lima, 2012). A desnutrição ocorre como resposta ao trauma cirúrgico e ao stress associado a este, com consequências na diminuição da resposta imunológica, na cicatrização ineficiente e no surgimento de infeções (Isidro \& Lima, 2012). Assim, é importante a intervenção do Enfermeiro no sentido de adequar a dieta no período pós-operatório e suprimir as necessidades energéticas. Uma vez que esse período está frequentemente associado a disfunções gastrointestinais, tais com a náusea e vómitos, a educação para a saúde tem um papel preponderante na sensibilização para a necessidade de uma dieta fracionada com evolução para uma dieta normal, consoante a tolerância (Grass et al., 2017).

Para a promoção precoce da autonomia, a mobilização precoce, que se inicia após a estabilização das alterações fisiológicas, engloba intervenções que se estima prevenirem o desenvolvimento de TVP, que incluem atividades tais como: alternância de posicionamentos; técnicas de mobilização articular e muscular; levante para a beira do leito; ortostatismo; transferência para a cadeira; deambulação (Nascimento et al., 2019; Viviani et al., 2019). Estas intervenções encontram-se igualmente discriminadas nos estudos de Kang et al. (2019); Yayla \& Özer (2019); Pu, Jiang, Leng \& Yang (2017); Jia et al. (2013). As atividades referidas visam reduzir os efeitos sobre $o$ sistema musculoesquelético, mantendo a funcionalidade da articulação e revertendo a fraqueza muscular, assim como reduzir o tempo de internamento e, consequentemente, os custos associados a este (Nascimento et al., 2019). Este dado corrobora os resultados obtidos por Kang et al. (2019), que pela implementação da mobilização precoce, teve 
repercussões positivas na ausência de complicações clínicas, assim como um menor tempo de permanência hospitalar e, por isso, um retorno mais rápido do paciente às suas atividades de vida diária. No estudo de Yayla \& Özer (2019), é também verificada uma diminuição significativa do desenvolvimento de complicações tardias pela aplicação da mesma intervenção.

Adicionalmente, numa revisão de Viviani et al. (2019), é exposto que a associação de diversas intervenções não farmacológicas pode diminuir em metade o risco de ocorrência de TVP. Na mesma revisão é ainda apresentado que a associação da mobilização ativa dos membros inferiores, dos exercícios respiratórios e, quando possível, da deambulação precoce reduz a taxa de ocorrência de complicações, nomeadamente a TVP. Indo de encontro ao já referido, no estudo de Yayla \& Özer (2019) foram também executados exercícios respiratórios profundos e promoção da tosse, bem como associados posicionamentos promotores da expansão da caixa torácica e da permeabilidade das vias aéreas. Um dos fatores que está associado à imobilidade no período pós-operatório é a cateterização vesical. Num estudo dirigido por Safdar et al. (2016), 25\% dos pacientes inquiridos associam à algaliação a sensação de limitação ou restrição da mobilidade. Além disso, a remoção precoce do cateter urinário demostrou reduzir o desconforto, possibilitar a mobilização precoce e diminuir o número de infeções associadas à cateterização vesical, que são contribuições importantes para a redução do tempo de permanência hospitalar (Roberts, Patel \& Smith, 2017). Assim, pode-se concluir que a movimentação ativa e passiva assistida e a deambulação precoce, por favorecerem o retorno venoso e evitarem a estase sanguínea, estão indicadas para pacientes com potencial desenvolvimento de processos trombóticos, em todas as estratificações de risco e, em especial, em situações de predisposição a hemorragia secundária à profilaxia farmacológica. (Costa, 2017). Outra das áreas de intervenção do enfermeiro na prevenção da TVP é a estimulação do retorno venoso, tal como se verificou no estudo de Pu, Jiang, Leng \& Yang (2017). Na realização de massagem dos membros inferiores é aplicada pressão no membro, contribuindo para a prevenção da TVP através do aumento da velocidade e do fluxo de sangue venoso. Numa investigação de Imai et al. (2017), que associa massagem da perna com mobilização passiva do tornozelo, comprovou-se que a incidência de TVP foi significativamente menor quando aplicadas essas terapias, em comparação com a não aplicação de qualquer medida preventiva. Esta intervenção demonstrou também ser benéfica ao aumento da fibrinólise e à ativação dos fatores de coagulação e vantajosa pela possibilidade da sua realização ser precoce, imediatamente após a cirurgia. De igual forma, a terapia por compressão pneumática intermitente tem como objetivo promover a circulação sanguínea do membro inferior e aumentar o fluxo venoso nas veias profundas do mesmo, reduzindo o risco de ocorrência de trombose. Num estudo realizado por Hou et al. (2017), verificou-se redução significativa da incidência de TVP num grupo submetido a terapia por compressão pneumática intermitente. Assim, a realização de massagem dos membros inferiores, bem como o uso de meias elásticas de compressão graduada e de dispositivos de compressão pneumática intermitente, ao exercerem pressão ao longo de todo o membro, reduzem a acumulação de sangue nas veias profundas, evitando a 
estase sanguínea, e aumentam a velocidade do fluxo sanguíneo com orientação do retorno venoso para o coração (Costa, 2017). Em adição às medidas já abordadas, a prevenção da ocorrência de TVP compreende também uma área totalmente interdependente que envolve a administração de terapêutica, nomeadamente de anticoagulantes. A terapêutica anticoagulante pode ser realizada com recurso a heparina não fracionada, a HBPM - como foi efetuado nos estudos de Pu, Jiang, Leng \& Yang (2017); Ghelase et al. (2013) - ou utilizando anticoagulantes orais, como o AAS - estudo de Pu, Jiang, Leng \& Yang (2017). Os anticoagulantes são indispensáveis quer no tratamento de trombos arteriais e venosos, quer em pacientes que foram submetidos a certas cirurgias, bem como no sentido de prevenir a ocorrência de TVP (Costa, 2017).

De acordo com Hou et al. (2017), o tratamento com recurso a heparina não fracionada e o tratamento com recurso a HBPM demonstram ter a mesma eficácia na prevenção de TVP. No mesmo estudo, verificou-se uma redução significativa da incidência de TVP num grupo submetido a tratamento com heparina de baixo peso molecular. Estes dados são consonantes com os obtidos no estudo de Ghelase et al. (2013), reforçando a importância da tromboprofilaxia através da administração de HBPM.

Também como profilaxia medicamentosa antitrombótica pode-se recorrer à administração de AAS. Num estudo de Lins et al. (2017) em que foi administrado AAS a pacientes em pós-operatório, foi observada uma incidência de TVP de apenas $2,7 \%$ no 6o dia do pós-operatório, assim como na 6a semana. Foi ainda verificada a inexistência de episódios de hemorragia, o que confere segurança ao uso deste fármaco como uma medicação profilática no período pós-operatório. Neste estudo recomenda-se o uso de AAS como profilaxia medicamentosa de TVP.

\section{CONCLUSÃO}

De acordo com o conteúdo exposto, pode-se concluir que a intervenção do Enfermeiro é crucial na prevenção de TVP através da realização de educação para a saúde, da promoção precoce da autonomia, da adequação do regime dietético, da estimulação do retorno venoso e da administração de terapêutica. Destas áreas de intervenção de Enfermagem, destacam-se os seguintes cuidados: ensinos sobre a prática de exercícios para os membros inferiores; ensinos no pós-operatório sobre mobilização precoce; realização de exercícios respiratórios profundos e de tosse; elevação da cabeceira entre 30 e 45; posição de sentado na cadeira ou cadeirão; realização de percursos de deambulação; massagem dos membros inferiores; aplicação de meias de compressão; administração de HBPM e AAS.

Assim, considera-se que o Enfermeiro deve utilizar as evidências científicas nesta área e desenvolver competências que lhe permitam estruturar e dirigir a intervenção a cada paciente, adequando cada cuidado face às necessidades que o mesmo apresenta. Além disto, foi evidente a necessidade de que as instituições de saúde disponham de um protocolo de atuação que vise prevenir o desenvolvimento de TVP.

No que diz respeito às limitações deste estudo, verificou-se um número reduzido de estudos sobre a temática direcionados para a prática da Enfermagem, o que evidencia a importância de um maior investimento científico na área. Também o facto de se ter efetuado uma limitação temporal à priori, bem 
como o facto de se terem excluído os artigos que não apresentavam o texto integral, limita-nos a garantia de que não existam outras publicações sobre a temática. Com os resultados obtidos, e com a análise efetuada, verifica-se que são relatadas diversas intervenções descritas como preventivas da ocorrência de TVP, no entanto, para determinar a sua real eficácia, é necessária a realização de estudos que efetuem esta análise. Assim, julga-se que devem ser realizados estudos que abordem as intervenções isoladamente, de forma a averiguar a influência de cada uma em específico na prevenção da TVP, sustentando a intervenção de Enfermagem.

\section{REFERÊNCIAS BIBLIOGRÁFICAS}

Amaral, C., Reis, J., Guimarães, L., Sá, A. C., Moreto, A., Araújo, F., Guimarães, M., Felicíssimo, P., Teixeira, J., Fonseca, C., Miranda, L. (2014). Recomendações Perioperatórias para Profilaxia do Tromboembolismo Venoso no Doente Adulto. Consenso Nacional Multidisciplinar 2014. Revista Da Sociedade Portuguesa De Anestesiologia, 23(3), 62-75. doi: 10.25751/rspa.4831

Barnard, N. D. (2018). Deep Venous Thrombosis. Nutrition Guide for Clinicians, 3, 1-4. Disponível em https://nutritionguide.pcrm.org/nutritionguide/view/ Nutrition_Guide_for_Clinicians/1342012/all/ Deep_Venous_Thrombosis

Bower, A. \& Royse, C. (2016). The importance of postoperative quality of recovery: influences, assessment, and clinical and prognostic implications. Canadian Journal of Anesthesia, 63, 176-183. DOI 10.1007/s12630-015-0508-7

Corrêa, R., Campos, F. \& Mancunzo, E. (2015). Hipertensão pulmonar tromboembólica crônica: tratamento medicamentoso dos pacientes não cirúrgicos. Pulmão RJ, 24(2), 55-60. Disponível em http://www.sopterj.com.br/wp-

content/themes/_sopterj_redesign_2017/_revista/20 15/n_02/full.pdf

Costa, Carla. (2017). Medidas preventivas do tromboembolismo venoso no doente hospitalizado: uma revisão integrativa da literatura.
Disponível em https://repositorio.ipv.pt/bitstream/10400.19/4585/1 /CarlalsabelSantosCosta\%20DM.pdf

Di Nisio, M., van Es, N. \& Büller, H. (2016). Deep vein thrombosis and pulmonary embolism. Lancet, 388(10063), 3060-3073. doi: 10.1016/S01406736(16)30514-1

AESOP. (2012). Enfermagem Perioperatória: da filosofia à prática de cuidados. Lisboa: Lusodidacta. ISBN: 978-972-8930-16-5.

Ghelase, M., Borugă, A., Râmboiu, S., Rotaru, A., Mărgăritescu, D., Cârţu, D. \& Ghelase, F. (2013). Study of the risk factors and prevention ofvenous thromboembolism in surgery. Current Health Sciences Journal, 39(1), 48-52. doi: 10.12865/CHSJ.39.01.09

Grass, F., Schäfer, M., Demartines, N. \& Hübner, M. (2017). Normal Diet within Two Postoperative DaysRealistic or Too Ambitious?. Nutrients, 9(12), 1336. https://doi.org/10.3390/nu9121336

Greenall, R. (2016). Using patient education to reduce risk of VTE. Nursing Times, 112(3), 3-6. Disponível em https://www.nursingtimes.net/clinical-

archive/patient-safety/using-patient-education-toreduce-risk-of-vte-11-07-2016/

Guo, M., Lu, L., Sun, Y., Li, L., Wu, M. \& Lang, J. (2019). Comprehensive functional exercises with patient education for the prevention of venous thrombosis after major gynecologic surgery: A randomized controlled study. Thrombosis Research, 178, 69-74. https://doi.org/10.1016/j.thromres.2019.04.013

Heil, J., Miesbach, W., Vogl, T., Bechstein, W., Reinisch, A. (2017). Deep Vein Thrombosis of the Upper Extremity. Deutsches Ärzteblatt International, 114(14), 244-249. doi: 10.3238/arztebl.2017.0244

Hou, D., Wang, Y., Jiang, W., Zuo, D. \& Sun, M. (2017). Efficacy and safety of low molecular weight heparin treatment, intermittent pneumatic compression therapy passive ankle exercise and nursing care in preventing deep venous thrombosis of the lower extremity following varicose vein surgery. Int I Clin Exp Med, 10(6), 9546-9554. ISSN:19405901/IJCEM0050961

Imai. N., Ito, T., Suda, K., Miyasaka, D. \& Endo, N. (2017). Manual calf massage and passive ankle motion reduce the incidence of deep vein thromboembolism after total hip arthroplasty. Journal of Orthopaedic of Science, 22(4), 726-730. http://dx.doi.org/10.1016/j.jos.2017.03.006 
Isidro, M. \& Lima, D. (2012). Adequação calóricoproteica da terapia nutricional enteral em pacientes cirúrgicos. Revista da Associação Médica Brasileira, 58(5), 580-586. https://doi.org/10.1590/S010442302012000500016

Jia, Y., Jin, G., Guo, S., Gu, B., Jin, Z., Gao, X., Li, Z. (2013). Fast-track surgery decreases the incidence of postoperative delirium and other complications in elderly patients with colorectal carcinoma. Langenbecks Arch Surg, 399(1), 77-84. doi: 10.1007/s00423-013-1151-9

Kang, Y., Liu, J., Chen, H., Ding, W., Chen, J., Zhao, B. \& Yin, X. (2019). Enhanced recovery after surgery (ERAS) in elective intertrochanteric fracture patients result in reduced length of hospital stay (LOS) without compromising functional outcome. Journal of Orthopaedic Surgery and Reserac, 14(209), 1-7. Doi: 10.1186/s13018-019-1238-2

Kmet, L. M., Cook, L. S., \& Lee, R. C. (2004). Standard quality assessment criteria for evaluating primary research from a variety of fields. Alberta Heritage Foundation for Medical Research (AHFMR) AHFMR HTA Initiative \#13., (February), 1-28

Lins, R., Filho, E., Oliveira, F., Santos, S., Rolim, T. \& Kreimer, F. (2017). Estudo prospectivo da aspirina como profilaxia do tromboembolismo em artroplastia total do quadril. Acta Ortop Bras, 26(2), 86-90. DOI: http://dx.doi.org/10.1590/1413-

785220182602187265

Lopes, R., Castro, J., Nogueira, C., Braga, D., Gomes, J., Silva, R. \& Brandão, M. (2019). Complicações do pósoperatório imediato de cirurgia cardíaca eletiva: estudo transversal à luz de Roy. Revista de Enfermagem Referência, 22, 23-32, https://doi.org/10.12707/RIV19042

Macneill, S. \& Bagot, C. (2018). Prevention and treatment of venous thromboembolic disease. Disponível em https://www.prescriber.co.uk/article/prevention-andtreatment-of-venous-thromboembolic-disease/

Nascimento, A., Mendonça, A., Silva, J., Santos, N., Silva, J. \& Cavalcante, T. (2019). Atuação do fisioterapeuta na mobilização precoce para prevenção da trombose venosa profunda. Revista Movimenta, 13(1), 128-138. ISSN:1984-4298

Olaf, M. \& Cooney, R. (2017). Deep Venous Thrombosis. Emergency Medicine Clinics of North America, 35(4), 743-770. doi:

\subsection{6/j.emc.2017.06.003}

Pinho, N., Viegas, K. \& Caregnato, R. (2016). Papel do enfermeiro no período perioperatório para prevenção da trombose venosa profunda. Revista SOBECC, 21(1), 28-36. doi: 10.5327/Z1414-4425201600010005

Pu, H., Jiang, H., Leng, Z., Yang, X. (2017). Investigation on the early-stage nursing intervention for deep venous thrombosis in traumatic fracture senile patients in perioperative period. Biomedical Research, 28(21), 9507-9510. ISSN 0970-938X

Roberts, S., Patel, K. \& Smith, S. (2017). Impact of avoiding post-operative urinary catheters on outcomes following colorectal resection in an ERAS programme: no IDUC and ERAS programes. ANZ Journal of Surgery, 88(5), 1-7. doi: 10.1111/ans.13916

Safdar, N., Codispoti, N., Purvis, S. \& Knobloch, M. (2016). Patient perspectives on indwelling urinary catheter use in the hospital. American Journal of Infection Control, 44(3), e23-e24. doi:10.1016/j.ajic.2015.10.011

Sociedade Portuguesa de Cirurgia. (2015). Tromboembolismo Venoso: Diagnóstico e Tratamento. Disponível em https://www.spcir.com/wpcontent/uploads/2016/06/Tromboembolismo_Venos o_Diagnostico_e_Tratamento_2015.pdf

Sousa, A., Bim, L., Hermann, P., Fronteira, I. \& Andrade, D. (2020). Complicações no pós-operatório tardio em pacientes cirúrgicos: revisão integrativa. Revista Brasileira de Enfermagem, 73(5), 1-7. http://dx.doi.org/10.1590/0034-7167-2019-0290

Sousa, L., Vieira, C., Severino, S., Antunes, A. (2017). A metodologia de revisão integrativa da literatura em enfermagem. Revista Investigação em Enfermagem, 17-26. Disponível em https://www.researchgate.net/publication/32131974 2_Metodologia_de_Revisao_Integrativa_da_Literatur a_em_Enfermagem

Viviani, A., Silva, M., Gomes, A., Molina, C. (2019). Aplicabilidade da mobilização precoce na prevenção de Trombose Venosa Profunda em ambiente hospitalar: Uma Revisão Sistemática. Revista Pesquisa em Fisioterapia, 9(3), 421-428. doi: 10.17267/22382704rpf.v9i3.2448

Yayla, A. \& Özer, N. (2019). Effects of early mobilization protocol performed after cardiac surgery on patient care outcomes. International Journal of Nursing Practice, 25(e12784), 1-11. doi: 10.1111/ijn.12784 